Supporting Information

\title{
A study on dislocation mechanisms of toughening in Cu-graphene
}

\section{nanolayered composite}

Subin Lee, Hadi Ghaffarian, Wonsik Kim, Taegu Lee, Seung Min Han, Seunghwa Ryu, and Sang Ho Oh

\section{Supplementary Figures}
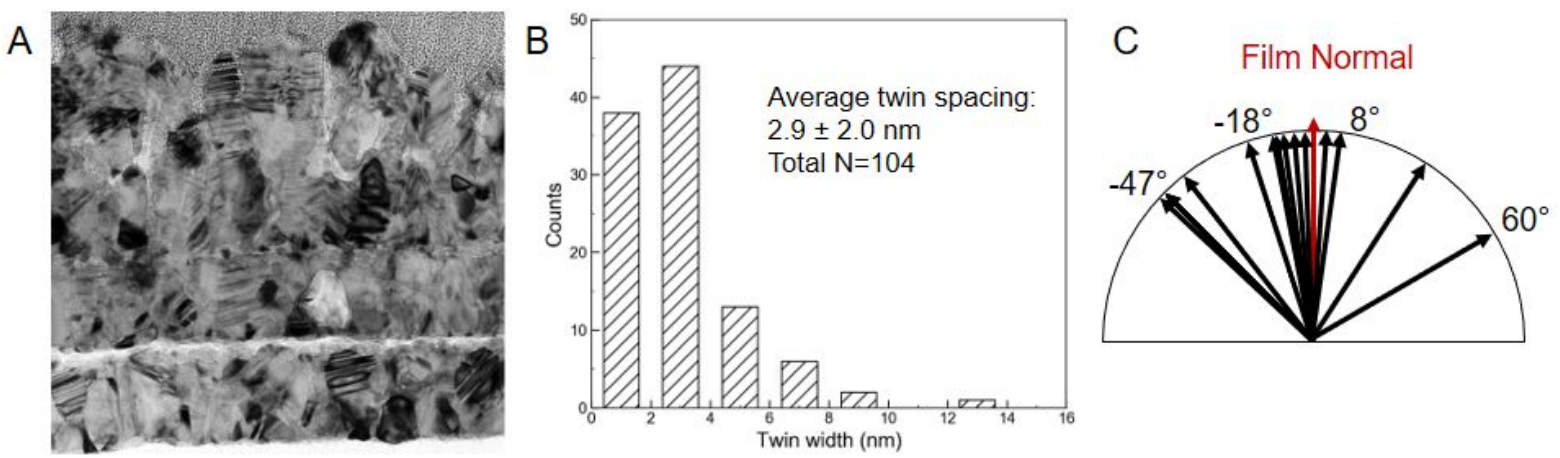

Figure S1. Microstructure and orientation of growth twins in $\mathrm{Cu}$. (a) A TEM bright field image shows a high density of twins in $\mathrm{Cu}$ grains. (b) Histogram of twin width measured from Figure S1A). The average width is $2.9 \mathrm{~nm}$ with a standard deviation of $2.0 \mathrm{~nm}$. (c) Orientation of twin boundaries with respect to the film normal direction. Majority of twins are parallel to the film normal direction. 
A
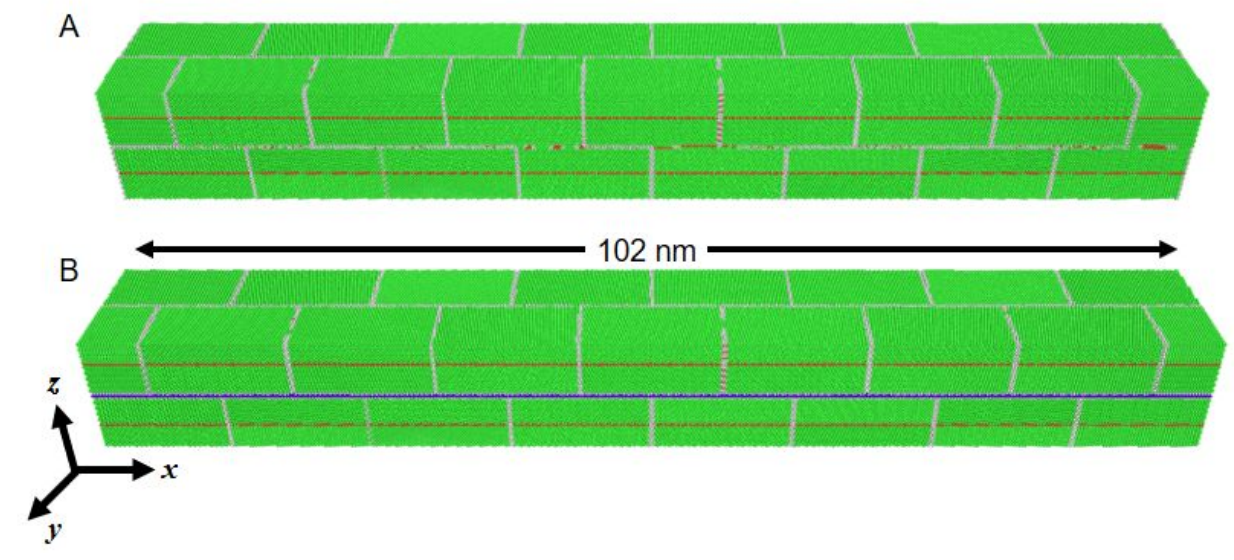
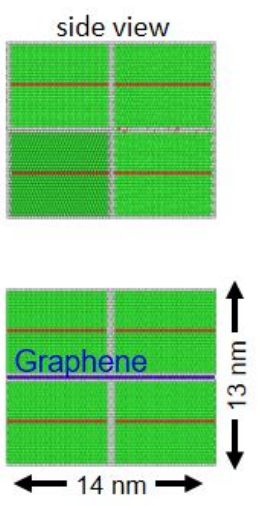

Figure S2. Constructed simulation samples for MD simulation of the uniaxial tensile test; (A) $\mathrm{Cu}$ bi-layer sample and (B) nanocomposite $\mathrm{Cu}$-graphene three-layer sample. Green, red, and white colors represent the $\mathrm{Cu}$ atoms with FCC structure, TBs, and GBs. Carbon atoms are colored blue. $\mathrm{Cu}$ grains are assembled considering an offset between the adjacent grains along $y$ - and $z$-directions. 

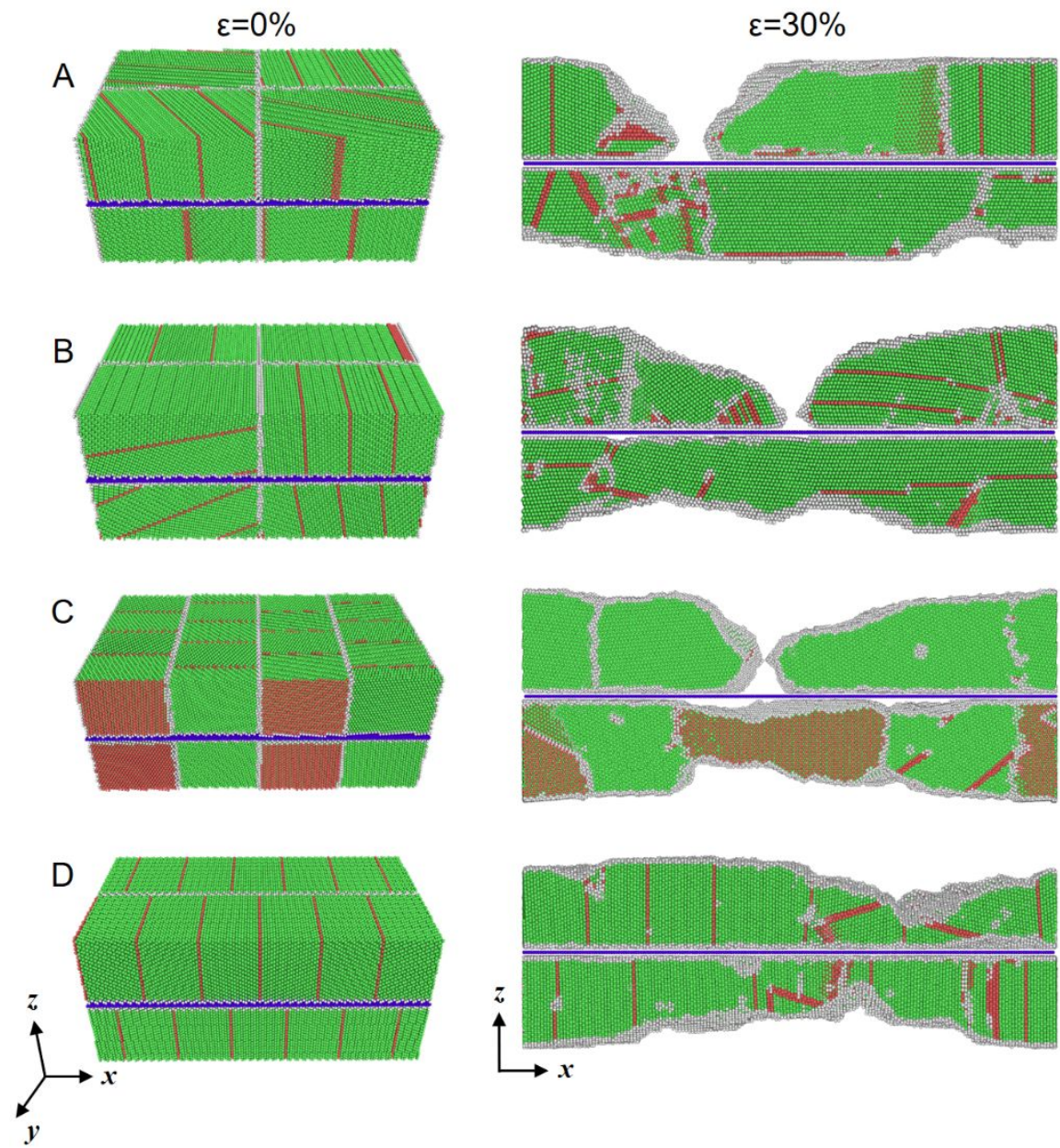

Figure S3. MD simulation results of tensile deformation behavior of nanocomposite $\mathrm{Cu}-$ graphene three-layer samples with different TB's orientation. TBs in (A) and (B) are parallel to the $z$ - and $y$-directions, respectively. TBs in (C) and (D) are parallel to $x-z$ and $y-z$ planes, respectively. Deformation results indicate that the interaction between the $\mathrm{Cu}$ and graphene layer is not affected by the crystal orientation of $\mathrm{Cu}$ layers. The higher ductility in (D) is attributed to the absence of GB perpendicular to the loading direction ( $x$-direction). Hence, the crack growth is confined along with the TB, whose strength is significantly higher than GBs. The Green and white colors represent the $\mathrm{Cu}$ atoms with FCC structure and GBs, respectively. The red color indicates both TBs and stacking faults. The carbon atoms are colored blue. 
Table S1. Material properties used in the crack phase field modeling.

\begin{tabular}{lcccc}
\hline Model & \multicolumn{2}{c}{ Cu-Gr } & \multicolumn{2}{c}{ Cu only } \\
\hline Property & $\mathrm{E}[\mathrm{GPa}]$ & $\mathrm{G}_{\mathrm{c}}\left[\mathrm{kN} \mathrm{mm}{ }^{-1}\right]$ & $\mathrm{E}[\mathrm{GPa}]$ & $\mathrm{G}_{\mathrm{c}}\left[\mathrm{kN} \mathrm{mm}{ }^{-1}\right]$ \\
\hline Soft & 128 & $2.048 \times 10^{-6}$ & 100 & $2.048 \times 10^{-6}$ \\
Soft-GB & 128 & $1.536 \times 10^{-6}$ & 100 & $1.536 \times 10^{-6}$ \\
Interface & 128 & $2.048 \times 10^{-8}$ & & \\
Stiff & 1000 & $1.6 \times 10^{-5}$ & & \\
Stiff-GB & 1000 & $1.2 \times 10^{-5}$ & & \\
\hline
\end{tabular}

\section{Experimental Methods}

\subsection{Fabrication of $\mathrm{Cu}$ - $\mathrm{Gr} \mathrm{ML}$ and $\mathrm{Cu} M L$ nanocomposite}

$\mathrm{Cu}-\mathrm{Gr} \mathrm{ML}$ and $\mathrm{Cu}$ ML nanocomposites have been fabricated by sputter deposition of $\mathrm{Cu}$ and roll-based dry transfer of graphene layers. For the fabrication of $\mathrm{Cu} \mathrm{ML}$, thickness of $100 \mathrm{~nm}$ of $\mathrm{Cu}$ layers have been sputter deposited 4 times for total composite thickness of $400 \mathrm{~nm}$ on a PI substrate. For the fabrication of $\mathrm{Cu}-\mathrm{Gr} \mathrm{ML}, \mathrm{CVD}$ grown single layer graphene is dry transferred onto $\mathrm{Cu}$ surface in between each deposition steps. Both $\mathrm{Cu} \mathrm{ML}$ and $\mathrm{Cu}-\mathrm{Gr} \mathrm{ML}$ nanocomposites have been post annealed at $250^{\circ} \mathrm{C}$ for 5 minutes using RTA.

\subsection{In situ TEM tensile straining}

TEM samples for in situ TEM straining tests were prepared by dual-beam focused ion beam (FIB, Helios Nanolab 600i, ThermoFisher). Carbon and platinum protective layer is used to protect thin films from the $\mathrm{Ga}^{+}$ion beam. $\mathrm{Ga}^{+}$ion beam with acceleration voltage of $30 \mathrm{kV}$ and $5 \mathrm{kV}$ were used for the thinning and final cleaning processes, respectively. To localize the applied strain effectively to the small volume of the sample, we used the site-specific FIB liftout method. ${ }^{1}$ A lamella with a size of $8 \mu \mathrm{m} \times 5 \mu \mathrm{m} \times 100 \mathrm{~nm}$ was glued on a custom made $\mathrm{Cu}$ straining grid which fits in to the TEM straining holder (Model 654, Gatan) by Pt deposition 
inside a FIB. Figure 2A shows a schematic drawing of the experimental setup for in situ TEM straining test. The central part of the $\mathrm{Cu}$ grid starts to deform by necking and simultaneously transfer the applied displacement to the FIB-prepared sample.

TEM imaging was conducted in bright-field imaging mode on aberration-corrected TEMs at an acceleration voltage of $300 \mathrm{kV}$ (Titan Themis, ThermoFisher). In situ tensile straining tests were carried out in the same microscopy by using a single tilt straining holder (Model 654, Gatan) and recorded with a high-speed camera at a frame rate of 50 frames per second and $2 \mathrm{k}$ $\times 2 \mathrm{k}$ resolution. The displacement was controlled by a step motor whose resolution is $1 \mu \mathrm{m}$ and the displacement rate was $1 \mu \mathrm{m} \mathrm{s}^{-1}$. No quantitative load-displacement data were obtained during the testing.

\subsection{Simulations and Modeling}

The MD simulation samples were prepared using the Matlab code by assembling different $\mathrm{Cu}$ thin layers as depicted in Figure S2. Each Cu layer was made by multiple cubic grains containing a twin boundary parallel to the $x-y$ plane located at the middle of the grains. The grains size was kept at $13 \times 7 \times 6 \mathrm{~nm}^{3}$ while their entire atoms were randomly rotated with respect to the normal direction ( $z$-direction). The $\mathrm{Cu}$ layers were then made by $8 \times 2$ duplications of $\mathrm{Cu}$ grains along $x$ - and $y$-directions, representing the observed columnar structure of $\mathrm{Cu}$ thin film at the nano-scale. The final samples were then constructed by assembling the $\mathrm{Cu}$ layers along the $z$-direction with or without the graphene layer at the middle. The dimension of constructed samples is $102 \times 14 \times 13 \mathrm{~nm}^{3}$ with $1,535,000$ and 1.593,000 atoms in $\mathrm{Cu}$ bi-layer sample and nanocomposite $\mathrm{Cu}$-graphene sample, respectively. For the nanocomposite $\mathrm{Cu}$-graphene sample, we considered a zigzag-oriented graphene layer along the $x$-direction with $2.0 \AA$ interlayer spacing between the $\mathrm{Cu}$ layers and graphene sheet. 
We also considered periodic boundary conditions (PBC) along the $x$ - and $y$-directions. In contrast, the free surface condition was considered along the $z$-direction. To prevent alignment between the adjacent GBs, the grains were assembled considering an offset with the adjacent grains along $y$ - and $z$ - directions (See Figure S2 for more details).

We used the hybrid potential method to describe the complex atomic interactions in the $\mathrm{Cu}-\mathrm{C}$ system. The embedded atom-method (EAM) interatomic potential ${ }^{2}$ was employed to describe the interaction between $\mathrm{Cu}$ atoms. The interlayer atomic interaction of the graphene layer was described by the adaptive intermolecular reactive empirical bond-order (AIREBO) potential. ${ }^{3}$ In addition, 12-6 Lennard-Jones (L-J) potential with well depth (ع) equal to $19.996 \mathrm{meV}$ and equilibrium distance $(\sigma)$ equal to $3.225 \AA$ was also used to describe the weak interaction between the thin metal film and graphene layer, including $\mathrm{Cu}-\mathrm{C}$ interaction.

MD simulations were then performed using the LAMMPS software ${ }^{4}$ at $300 \mathrm{~K}$. Before the mechanical testing; samples were gradually heated to $300 \mathrm{~K}$ and equilibrated for $100 \mathrm{ps}$ under zero pressure with the Nosé-Hoover isobaric-isothermal (NPT) ensemble. To avoid thermal instability in the graphene layer, we used a stepwise heating procedure, including intermediate thermal equilibration for $25 \mathrm{ps}$ at 100, 200, and $300 \mathrm{~K}$. The uniaxial tensile load was then applied along the $\mathrm{x}$-direction with a constant strain rate of $5 \times 10^{8} \mathrm{~s}^{-1}$ up to 20 percent total strain. To mimic the observed deformation mode in the experiments, we fixed the sample's size along the y-direction, providing a typical plane strain deformation mode. We also used the common neighbor analysis (CNA) method $^{5,6}$ to visualize the local crystalline structure of each atom in $\mathrm{Cu}$ layers.

\subsection{Crack phase field Modeling}


The crack phase field modeling was performed to better elucidate the role of the weak interfaces on the crack path and enhancing toughness of the $\mathrm{Cu}-\mathrm{Gr}$ composite. The crack phase field modeling describes the crack propagation through a diffusive crack topology and the phase which is a scalar variable from 0 to 1 means the degree of failure. ${ }^{7,8}$ In this study, we used the crack phase-field model based on the hybrid formulation implemented by Jeong et $a l^{9}$ via the User Element (UEL) function in the commercial finite element method (FEM) solver, Abaqus/Standard. We note that for, the ease of implementation, the crack phase modeling can only describe the linear brittle materials and is not intended to offer the accurate prediction on the crack-induced plasticity. Still, the crack phase field model can qualitatively model the change in the crack propagation path due to the presence of the weak $\mathrm{Cu}-\mathrm{Gr}$ interface. The geometries of both multilayer structures in Figure S3 are expressed in micrometer units. The input parameters are the elastic modulus $(E)$ and surface energy release rate $\left(G_{c}\right)$, as summarized in Table S1. We set soft and stiff materials to represent copper and graphene, respectively, and set other regions to represent a few different interfaces such as copper grain boundary, graphene grain boundary, and copper-graphene interface. The mechanical properties of the stiff material were taken from previous experimental study of graphene ${ }^{10}$, while the modulus of the soft material is chosen from experimental study of copper. ${ }^{11}$ Instead of experimentally measured $G_{c}$ value of copper, we empirically assigned the $G_{c}$ of the soft material and soft grain boundary regions such that only intergranular crack propagation occurs. Each grain boundary was assumed to have the same elastic modulus with each material, but $75 \%$ of the surface energy release rate. Finally, the weak copper-graphene interface region was set to have an identical elastic modulus but 1/100 lower surface energy release rate with the copper region, in order to represent the difference between van der Waals interaction strength and covalent bonding strength. 
(1) Li, C.; Habler, G.; Baldwin, L. C.; Abart, R. An Improved FIB Sample Preparation Technique for Site-Specific Plan-View Specimens: A New Cutting Geometry. Ultramicroscopy 2018, 184, 310-317.

(2) Mishin, Y.; Mehl, M. J.; Papaconstantopoulos, D. A.; Voter, A. F.; Kress, J. D. Structural Stability and Lattice Defects in Copper: Ab Initio, Tight-Binding, and Embedded-Atom Calculations. Physical Review B - Condensed Matter and Materials Physics 2001, 63 (22), 2241061-22410616. https://doi.org/10.1103/PhysRevB.63.224106.

(3) Brenner, D. W.; Shenderova, O. A.; Harrison, J. A.; Stuart, S. J.; Ni, B.; Sinnott, S. B. A Second-Generation Reactive Empirical Bond Order (REBO) Potential Energy Expression for Hydrocarbons. Journal of Physics Condensed Matter 2002, 14 (4), $783-$ 802. https://doi.org/10.1088/0953-8984/14/4/312.

(4) Plimpton, S. Fast Parallel Algorithms for Short-Range Molecular Dynamics. Journal of Computational Physics. 1995, pp 1-19. https://doi.org/10.1006/jcph.1995.1039.

(5) Honeycutt, J. Dana.; Andersen, H. C. Molecular Dynamics Study of Melting and Freezing of Small Lennard-Jones Clusters. The Journal of Physical Chemistry 1987, 91 (19), 4950-4963. https://doi.org/10.1021/j100303a014.

(6) Faken, D.; Jonsson, H. Systematic Analysis of Local Atomic Structure Combined with 3D Computer Graphics. Computational Materials Science 1994, 2 (2), 279-286. https://doi.org/10.1016/0927-0256(94)90109-0.

(7) Msekh, M. A.; Sargado, J. M.; Jamshidian, M.; Areias, P. M.; Rabczuk, T. Abaqus Implementation of Phase-Field Model for Brittle Fracture. Computational Materials Science 2015, 96, 472-484. https://doi.org/10.1016/j.commatsci.2014.05.071.

(8) Molnár, G.; Gravouil, A. 2D and 3D Abaqus Implementation of a Robust Staggered Phase-Field Solution for Modeling Brittle Fracture. Finite Elements in Analysis and Design 2017, 130, 27-38. https://doi.org/10.1016/j.finel.2017.03.002.

(9) Jeong, H.; Signetti, S.; Han, T.-S.; Ryu, S. Phase Field Modeling of Crack Propagation under Combined Shear and Tensile Loading with Hybrid Formulation. Computational Materials Science 2018, 155, 483-492. https://doi.org/10.1016/j.commatsci.2018.09.021.

(10) Zhang, P.; Ma, L.; Fan, F.; Zeng, Z.; Peng, C.; Loya, P. E.; Liu, Z.; Gong, Y.; Zhang, J.; Zhang, X.; Ajayan, P. M.; Zhu, T.; Lou, J. Fracture Toughness of Graphene. Nat Commun 2014, 5 (1), 3782. https://doi.org/10.1038/ncomms4782.

(11) Dolbow, J.; Gosz, M. Effect of Out-of-Plane Properties of a Polyimide Film on the Stress Fields in Microelectronic Structures. Mechanics of Materials 1996, 23 (4), 311321. https://doi.org/10.1016/0167-6636(96)00021-X. 